\title{
Limitations of the modelling of crack propagating through heterogeneous material using a phase field approach
}

\author{
Hervé Henry \\ Laboratoire Physique de La Matière condensée, École Polytechnique, CNRS, 91128 \\ Palaiseau Cedex, France
}

\begin{abstract}
The modeling of crack propagation in a heterogeneous material using a phase field model is studied numerically in a simple test case: the crack meets a wedge of higher fracture energy. It is shown that when the crack cannot enter the wedge, phase field results are in qualitative agreement with theoretical predictions with moderate quantitative discrepancies. When the crack can propagate in both regions, numerical results show that the interplay of diffuse interface modelling used in the phase field model with the interface between the two regions induces spurious effects that are unphysical.
\end{abstract}

\section{Introduction}

Heterogeneous materials are widespread in engineering and material science and they include charged polymers, polymer blends after phase separation, concrete, composite materials, alloys and biomaterials such as nacre or metamaterials [9, 25, 1]. Their mechanical properties such as their elastic coefficients can be computed using various strategies that rely on the use of representative volume elements. However, the understanding of their resistance to failure is limited. This is due to the fact that a crack propagating in a heterogeneous material under a simple loading will have a complex shape that cannot be predicted easily since it is the result of the interplay of the fracture propagation laws and the microstructure of the material. When considering a heterogeneous material, homogenization approaches that use experimentally determined quantities such as fracture energy can be made. However, one cannot predict crack properties for a given microstructure from the properties of the phases that compose the material. As a result, designing the microtructure of the phases that compose a material to reach given fracture energies [9, 25, 1] is impossible and a model that would allow such computation is highly desirable.

In homogeneous material the Linear Elastic Fracture Mechanics theory (LEFM) is efficient[11] when describing the propagation of a single crack in 2D materials. In the case of heterogeneous material, predictions made using the LEFM[26, 13. 
are possible. But in general, the use of this approach is difficult due to the complex crack paths that are present. Moreover the extension of LEFM to homogeneous 3D systems is difficult. Therefore, in the case of $3 \mathrm{D}$ heterogeneous material, the application of LEFM theory is likely to prove extremely difficult. Among the different models of crack propagation, the phase field model could describe correctly failure of heterogeneous material because of its ability to describe complex crack paths in both $2 \mathrm{D}$ and $3 \mathrm{D}$,

Indeed, the phase field model of crack propagation [19, 10, 4, 16], has been widely used to study crack propagation in homogeneous material and has been shown to be an effective way of describing crack propagation in various contexts. It has been shown that there is a very good quantitative agreement between the phase field model, the LEFM and more importantly well controlled experiments in the case of complex crack paths such as the oscillating cracks in a thermal gradient 38, 32, 33, 7] and the relaxation of crack fronts under mixed mode loading (I+III) 34, 31, 17, 6, 23. Since in the phase field model the fracture energy is a function of the parameters of the coupled partial differential equations it can describe a crack propagating in a heterogeneous material where the fracture energy is varying slowly (on large lengthscales comapred to the process zone size) by adding slow spatial variations of the model parameters. Such an approach has been used to model variations of the fracture energy anisotropy [12, 24, 30] or of the fracture energy [21, 18. It is then tempting to consider a crack propagating in a heterogeneous material that consists of homogeneous regions with different fracture energies that are separated by sharp interfaces.

Some preliminary simulations have shown results that are apparently qualitatively correct [36, 18. In the following I present numerical simulation results that were performed in a simple test geometry. These results indicate that in such a setup, the interface thickness between the two regions $\left(w_{\text {int }}\right)$ has dramatic effects on the numerical results unless it is small when compared to the phase field internal length $\left(w_{\varphi}\right)$. Furthermore, even when the interface thickness seems to be small enough to ensure independence of the crack propagation regime, results are unphysical. As a result, one should take extreme care when considering crack propagation in heterogeneous material in the presence of interfaces. The paper is organized as follows. First the phase field model is rapidly described and the mathematical description of the heterogeneities is given. Thereafter, the numerical setup and the geometry that has been studied are presented. This is followed by a description of the numerical results. Finally the discussion focuses on the questions that arise from the results and on a comparison with theoretical work relying on the LEFM theory.

\section{Phase Field Model and numerical implementation}

While having distinct theoretical bases ranging from the regularization of free discontinuities [10] to a phenomenological approach 19] inspired by phase transitions, the so called phase field models of crack propagation share the same basic idea. A free energy functional is written and the system evolves toward a minimum (local or global) of this functional. The evolution toward 
the minimum can be the result of a minimization of the free energy with respect to both the strain field and the phase field with, usually some constraints such as irreversibility. It can also be a relaxation equation for the phase field coupled to the wave equation (with possibly a dissipative term) for the strain field. In all cases it ensures the decrease of the free energy functional:

$$
\mathcal{F}=\int\left(\left(\frac{D}{2}|\nabla \varphi|^{2}+h V(\varphi)-\varepsilon_{c} g(\varphi)\right)+g(\varphi)\left(\frac{\lambda}{2}(\operatorname{tr} \varepsilon)^{2}+\mu \operatorname{tr}\left(\varepsilon^{2}\right)\right)\right) \mathbf{d} \mathbf{x}
$$

where $\lambda$ and $\mu$ are the Lamé coefficients and $\varepsilon$ is the strain tensor. The function that relates the phase field to the local elastic moduli is $g$. Here, the phase field $\varphi$ goes from 1 (undamaged) to 0 , totally broken and therefore $g$ ranges from 0 for $\varphi=0$ to 1 for $\varphi=1$. This choice corresponds to that made in [19. It ensures, as was proven in 19 that in the limit of vanishing interface thickness, the equilibrium solution converges toward a traction free crack. The potential $h V-\varepsilon_{c} g$ can either be a tilted double well [19] (tilted by $\varepsilon_{c}$ ) or a single well potential [10. It can also be a single obstacle potential, as in [36. All these choices lead to the same qualitatively correct behaviour in 2D and yield very similar results when describing the propagation of a single crack [22]. Here, following [19], the coupling function $g$ has been chosen to be

$$
g(\varphi)=4 \varphi^{3}-3 \varphi^{4}
$$

so that the $1 \mathrm{D}$ equilibrium crack solution corresponds to a situation where the residual stress in the unbroken material vanishes. The potential $h V$ is equal to $h(1-\varphi)^{2} \varphi^{2}$. With such a choice, the actual potential in the model of [19] is $h V(\varphi)-\varepsilon_{c} g(\varphi)$, which, since the coupling function has a zero slope in $\varphi=1$ leads to a purely elastic unbroken phase 22] similarly to what is observed when considering single obstacle type models 36, 37, 35. Here we have chosen $h=1$, $D=1$ and $\varepsilon_{c}=1$.

An important difference between the single obstacle model and the double well model is the way crack nucleation occurs. This will be qualitatively discussed now. In the single obstacle model [37, 22, the nucleation occurs as soon as the load is such that the elastic energy density is above the propagation threshold (which, with notations used here, is equal to $\varepsilon_{c}$ if interface curvature effects are neglected.) in a large enough region (where large enough is above a few material lengthscales $\left.D /\left(h, \varepsilon_{c}\right)\right)$. In the double well model, the elastic energy density should be above another threshold (that is larger by $\approx h / \varepsilon_{c}$ ) in a large enough region. Hence, in the single obstacle model a crack is likely to nucleate in a domain where the elastic energy density is below the propagation threshold almost everywhere. On the other hand, in the double well potential when a crack nucleates, it is likely that the elastic energy density is above the propagation threshold in a macroscopic domain around the nucleation site 1 . Therefore the two models will probably exhibit dramatically different post

${ }^{1}$ For a discussion on a system similar to cracks see 8 
nucleation behaviour. In the same spirit, the behaviour when the crack propagates under extremely high loads (close to material strength) should be different. Here, since nucleation is not studied this difference between the models can be safely ignored. In the same spirit, the fact that nucleation is not considered here, implies that the description of the crack propagation by the phase field model is correct when the phase field length scale $w_{\varphi}$ is much smaller than the macroscopic lengthscale, and when the phase field model parameters give the correct fracture energy. This is indeed confirmed by simulations performed (not shown) using the single obstacle model used in [37.

The evolution equations for the phase field and the displacement field are:

$$
\begin{aligned}
\partial_{t} \varphi & =-\beta p\left(\operatorname{tr} \varepsilon, \frac{\delta \mathcal{F}}{\delta \varphi}\right)\left(\frac{\delta \mathcal{F}}{\delta \varphi}\right) \\
& =-\beta p\left(\operatorname{tr} \varepsilon, \frac{\delta \mathcal{F}}{\delta \varphi}\right)\left(D \Delta \varphi-h V^{\prime}(\varphi)+\varepsilon_{c} g^{\prime}(\varphi)-g^{\prime}(\varphi)\left(\frac{\lambda}{2}(\operatorname{tr} \varepsilon)^{2}+\mu \operatorname{tr}\left(\varepsilon^{2}\right)\right)(4)\right. \\
\rho \partial_{t t} u_{i} & =-\frac{\delta \mathcal{F}}{\delta u_{i}}+D_{\text {diss }} \Delta \dot{u}_{i}
\end{aligned}
$$

where $\rho$ is the mass density equal to $1, \beta p$ is a kinetic parameter that has three purposes:

- $\beta$ is proportional to the energy dissipation in the process zone [14.

- $p$ ensures crack irreversibility: if

$$
\frac{\delta \mathcal{F}}{\delta \varphi}<0
$$

then $p=0$

- $p$ distinguishes between compression and extension if $\frac{\delta \mathcal{F}}{\delta \varphi}>0$ : if $\operatorname{tr} \varepsilon>$ 0 (i.e. under extension) then $p=1$. On the contrary if $\operatorname{tr} \varepsilon<0$ (i.e. under local compression), $p$ is a positiv $\phi^{2}$ number chosen so that $\partial_{t} \varphi$ is the minimum of 0 (to keep irreversibility and avoid crack healing under compression) and

$$
-\beta\left(D \Delta \varphi-h V^{\prime}(\varphi)+\varepsilon_{c} g^{\prime}(\varphi)-g^{\prime}(\varphi)\left(\frac{\lambda-K_{\text {Lame }}}{2}(\operatorname{tr} \varepsilon)^{2}+\mu \operatorname{tr}\left(\varepsilon^{2}\right)\right)\right)
$$

where $K_{\text {Lame }}=\lambda+2 \mu / 3$ is the bulk modulus.

While the first rule ensures energy dissipation at the crack tip, the two last one simply ensure crack irreversibility and the fact that energy due to compression does not participate to crack advance.

$D_{\text {diss }}$ is a fluid viscosity. Two limiting cases for $D_{\text {diss }}$ have been used here. In the first $D_{\text {diss }}=0.01$ is taken small to ensure a negligible dissipation during

${ }^{2}$ The fact that $p$ is positive ensures the decrease of $\mathcal{F}$. 


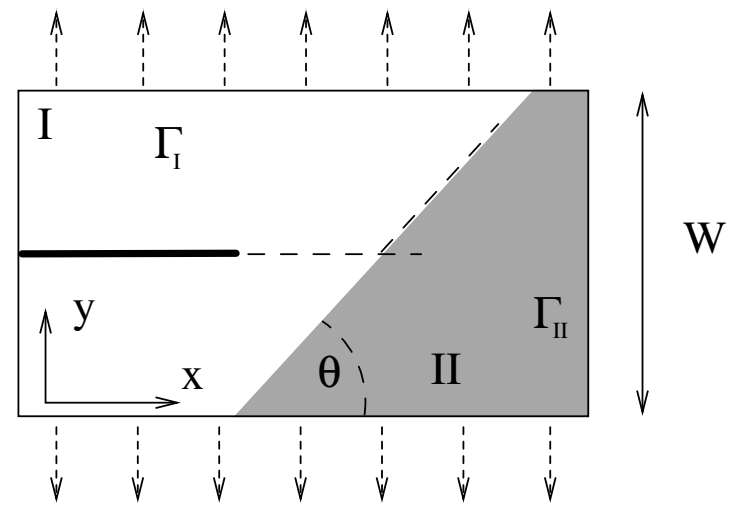

Figure 1: schematic view of the material, the initial crack and the coordinates. The fracture energies in white (resp. grey) regions are $\Gamma_{I}$ (resp. $\Gamma_{I I}$ ). The grey region is called the obstacle in the manuscript. The elastic boundary conditions are imposed displacement $u_{x}=0$ and $u_{y}= \pm \Delta_{y} / 2$ at $Y=\mp W / 2$ and at $x=0$ and $x=L u_{x}=0$ and $u_{y}=\Delta_{y}(y / W)$

wave propagation. The actual dynamic crack propagation is modeled with the possibility of branching events at high crack speed. [20, 14, 3, 15]. In the second it is chosen so that the relaxation toward mechanical equilibrium of the elastic field is fast so that the system mimics well quasistatic crack propagation $\left(D_{\text {diss }}=0.4\right)^{3}$. Here, it has been found that for a given load and fracture energy, the qualitative behaviour of crack (i.e. whether they propagate or not) along the interface with the obstacle (see fig. 11) is independent of the value of $D_{\text {diss }}$ in these two limiting cases.

This work is limited to the study of a crack interacting with a single inhomogeneity or obstacle which is characterized by a higher fracture energy than the matrix, while it has the same elastic coefficients. Such a choice is clearly unrealistic. However the purpose of this paper is to show the issues that arise from introducing interfaces between regions of different fracture energies in the phase field model. Therefore in order to avoid the additional complexity that will arise from heterogeneities in the elastic coefficients, they are taken equal in both phases. In the same spirit of simplification a pure mode I crack propagating in a homogeneous material until it reaches a flat interface with a region of higher fracture energy is considered as it can be seen in fig. 1. In the early work of Karma and coworkers[19], it was shown that in the phase field model, the fracture energy is:

$$
\Gamma=\int_{0}^{1}\left(\sqrt{D\left(\varepsilon_{c}(1-g(\varphi))+h V(\varphi)\right)}\right) d \varphi
$$

\footnotetext{
${ }^{3}$ When $D_{\text {diss }}$ is large relaxation is very slow since dissipation prevents a rapid motion of the material point, when $D_{\text {diss }}$ is small the oscillations are undamped and relaxation toward mechanical equilibrium is slow. 17. The value used here was determined by minimizing the relaxation time of an elastic sheet with a non moving crack toward equilibrium
} 
and is a function of $D>0, \varepsilon_{c}>0$ and $h \geq 0$. In $1 \mathrm{D}$, the exact shape of the phase field profile and the material lengthscale are also a function of these parameters. The exact interface profile shape (up to a rescaling by the material lengthscale) is a function of the respective magnitude of $h$ and $\varepsilon_{c}$ while for a given ratio $h / \varepsilon_{c}$, the material lengthscale $w_{\varphi}$ is proportional to $\sqrt{D / \varepsilon_{c}}$. Since this work is solely focused on the propagation of a crack along an obstacle it has been chosen to avoid the additional complexity of describing varying material lengthscales and interface profiles. To this purpose the free energy functional, following [19] is chosen:

$$
\begin{aligned}
\mathcal{F}= & \int\left((1+k(\mathbf{r}))\left(\frac{D}{2}|\nabla \varphi|^{2}+h V(\varphi)-\varepsilon_{c} g(\gamma)\right)+\right. \\
& \left.g(\varphi)\left(\frac{\lambda}{2}(\operatorname{tr} \varepsilon)^{2}+\mu \operatorname{tr}\left(\varepsilon^{2}\right)\right)\right) \mathbf{d x}
\end{aligned}
$$

With such an expression, the shape of the equilibrium solution for the fracture is independent of $k(\mathbf{r})$ and the fracture thickness $w_{\varphi}$ is kept constant while the local fracture energy of the simulated material is $\Gamma_{I I}=\Gamma_{I}(1+k(\mathbf{r}))$. As a result, the material strength in the region $\mathrm{II}$ is $(1+k(\mathbf{r}))$ times higher than in the region $\mathrm{I}\left[37\right.$. However, here, $w_{\varphi}$ is solely a regularization lengthscale and can be taken to be large when compared to actual material lengthscales as long as the following conditions are met: it is much smaller than macroscopic lengthscales and nucleation is not present.

Here, $k(\mathbf{r})$ is, using Cartesian coordinates

$$
\begin{aligned}
k(x, y) & =a_{0}\left(1+\tanh \left(d / w_{\text {int }}\right)\right) / 2 \text { with } \\
d & =\cos \theta\left(x-x_{o f f}\right)+\sin \theta(y)
\end{aligned}
$$

where $a_{0}>0$ is the amplitude of fracture energy increase, $d$ is the signed distance between a point $(x, y)$ and a straight line making an angle $\theta$ with the direction of propagation of the crack (see fig. 11). $w_{\text {int }}$ is the model parameter that measures the interface thickness between the regions I and II. For instance with $a_{0}=1$, the fracture energy is doubled when crossing the interface. In this context the crack is expected to either propagate along the interface, to stop or to propagate through the tougher region II. In the following we will present numerical results obtained with a phase field model of crack propagation in this setup and discuss whether they correspond to expected behaviours, keeping in mind that here the interface is a pure geometrical object that has no specific properties such as interface toughness.

These PDEs (3, 5) were simulated, using a finite difference scheme with a simple forward Euler time stepping scheme on a rectangular grid (see fig 1). The grid spacing $(\delta x=0.3)$ was chosen so that the phase field interface thickness (i.e. half the crack thickness) is approximately 4 grid points which ensures that lattice pinning can be neglected. In addition, simulations using a coarser grid and a finer grid $(\delta x=0.3 / \sqrt{2}$ and $\delta x=0.3 \sqrt{2})$ gave the same results with an error smaller than a few percents. The boundary conditions for the elastic field 

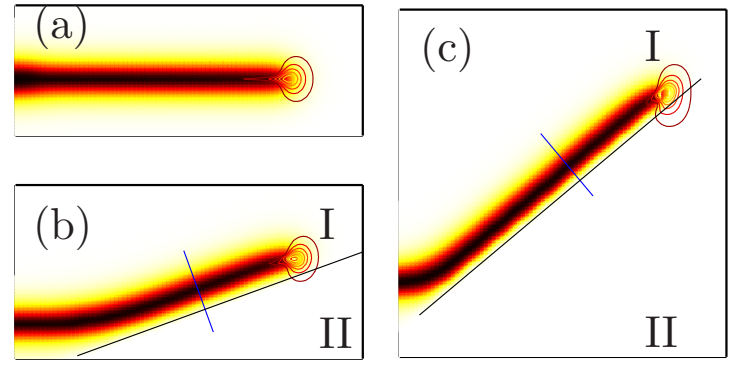

Figure 2: Contour plot of the elastic energy density at the end of a simulation for which the crack has stopped superimposed on the phase field color plot (white corresponds to $\varphi=1$ and black to $\varphi=0$. The straight black line corresponds to the boundary of the obstacle. The plots (a) corresponds to a freely propagating crack. Plot (b) to a stopped crack with $\theta=20^{\circ}$ and (c) to a stopped crack with $\theta=40^{\circ}$. It is worth noting that in the later case, the maximum of the elastic energy density is higher than in the case of a freely propagating crack. It is worth mentioning that the contour plot of the elastic energy density present a tail in the fully broken region that is due to the slow relaxation of the phase field toward its zero equilibrium value in the broken region. The phase field profile and along the blue lines are plotted in fig 4

are fixed displacements:

$$
u_{y}= \pm \Delta y / 2, \quad u_{x}=0 \text { at } y= \pm W / 2
$$

The elastic energy stored in the material is then $(\lambda / 2+\mu) \Delta y^{2} / W$ where $\lambda$ and $\mu$ are the Lamé coefficients. The system size $W$ was varied between 216 and 864 without any quantitative changes, when the elastic energy stored in the strained material was kept constant. This indicates that the ratio between interface thickness, the so called the internal length, of the phase field model and the system size does not affect the results. Therefore, it can be safely assumed that the simulatoions were performed in a limit for which the internal lengthscale is small enough to ensure that the model approximates well the infinitely sharp crack of the LEFM.

\section{Results}

\subsection{Infinitely tough region}

We first describe the propagation of a crack that meets an infinitely tough region where the phase field is set to 1 and does not change with time. In this case, the crack can only propagate along the interface between the normal medium and the infinitely tough medium. The questions that have to be answered are whether the crack propagates and at what velocity. Here we will mostly limit ourselves to the first since a theoretical prediction can be made.

To this end, we neglect the effect of the crack propagation velocity and suppose that the stress intensity factors before the kink are

$$
\left(K_{1}, K_{2}\right)=\left(A \Delta_{y}, 0\right)
$$



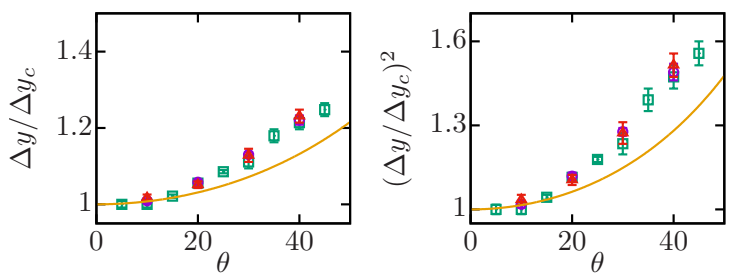

Figure 3: Left: Plot of the theoretical prediction of the threshold of propagation (solid line) together with the propagation threshold obtained from simulations. The system size was $W=W_{0}=$ (square), $W=2 W_{0}$ (circles) and $W=4 W_{0}$ (triangles). For all system sizes, $\Delta_{y}$ has been rescaled by the threshold value for propagation $\Delta y_{c}$ and error bars are given. Right: same plot using the fracture energy (i.e. $\left(\Delta y / \Delta y_{c}\right)^{2}$ instead of the applied displacement.

where $\mathrm{A}$ is a real constant that depends on the geometry of the material, and $\Delta_{y}$ is the applied displacement at the boundaries of the system. From this, using equations (2), (3) and (66) from [2, the stress intensity factors after deflection $\left(K_{1}\left(\theta, K_{0}\right), K_{2}\left(\theta, K_{0}\right)\right)$ can be computed as functions of the kink angle $\theta$ and of the stress intensity factor before the kink $K_{0}$. In our particular case where $K_{2}=0$ before the kink the SIFs after the kink write, using the notation of [2] at zeroth order in the length of the kinked crack :

$$
K_{1}=F_{11}(\theta) K_{0}, K_{2}=F_{21}(\theta) K_{0}
$$

where $F_{11}$ and $F_{21}$ are universal functions of $\theta$. From this the energy release rate associated with the crack propagation can be computed:

$$
G\left(K_{0}, \theta\right)=\left(K_{1}\left(\theta, K_{0}\right)\right)^{2}+\left(K_{2}\left(\theta, K_{0}\right)\right)^{2} .
$$

One expects the crack to propagate when $G\left(K_{0}, \theta\right)$ is larger than the fracture energy $\Gamma$ (which can be computed using the parameters of the phase field model[19]).

In order to have an estimate of $\Gamma$ that takes into account the possible errors induced by the numerical implementation, we compute the threshold value $\Delta_{y c}$ above which the straight crack starts to propagate, and from this we have: $\Gamma=\left(A \Delta_{y c}\right)^{2}$. As a result, one expect the crack to propagate as soon as $\left(K_{1}\left(\theta, K_{0}\right)\right)^{2}+\left(K_{2}\left(\theta, K_{0}\right)\right)^{2}$ is larger than $\left(A \Delta_{y c}\right)^{2}$. Taking into account equations 11, 12), this translates into

$$
\Delta_{y}^{2} \times\left(F_{11}(\theta)^{2}+F_{21}(\theta)^{2}\right)>\Delta_{y c}^{2},
$$

where the proportionality constant $A$ is no longer present.

In the situation described above the crack is forced either to kink or to stop propagating, so we expect that the threshold $\Delta_{y}$ above which the crack propagates along the interface to obbey the eq. (14). The result of simulations are now described and compared to the prediction of eq. (14).

As expected, two possible behaviours are observed : either the crack stops or it propagates along the interface. When the crack stops, due to inertial effects, 

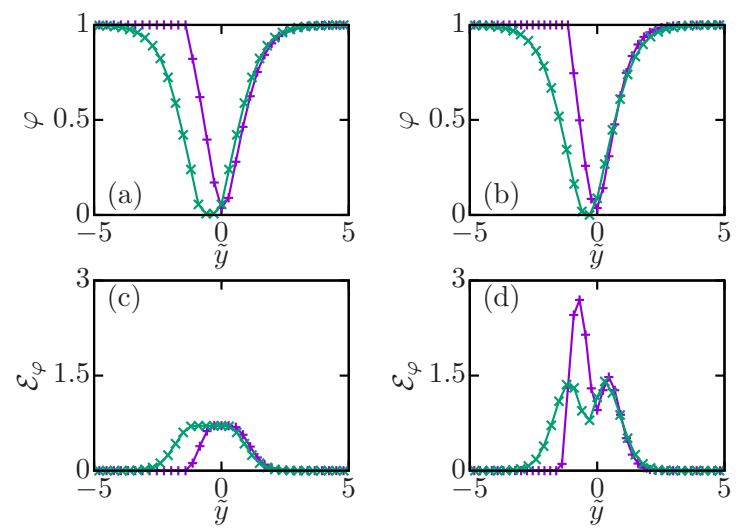

Figure 4: Solid lines: phase field profile perpendicular to the crack path before (green X) and after (purple + ) meeting the obstacle for $\theta=20^{\circ}(\mathrm{a})$ and $\theta=40^{\circ}(\mathrm{b})$. The distance from the crack midline is denoted $\tilde{y}$. The phase field energy density is plotted in (c) and (d). The line along which the profiles are plotted are represented in fig. 2

it propagates over a small distance along the interface before stopping. In figure 2. the crack interface profile corresponding to $\varphi=0.5$ is plotted together with the obstacle and the elastic energy density in the three cases (crack propagating freely, crack propagating along the interface and crack stopped after a short transient). In all three cases, the applied load $\Delta_{y}$ is the same.

The duration of the transient regime before the crack reaches a steady state (either stopped or propagating along the interface) and can be tuned by changing the amplitude of the dissipation terms. However the nature of the steady state itself is independent of that dissipation terms, and a comparison between theoretical prediction and numerical results can be made.

In figure 3 the prediction is plotted together with the results of the phase field simulations for angles ranging from $0^{\circ}$ to $45^{\circ}$ and three distinct system sizes, the phase field model parameters, being kept unchanged. First one must note that the simulations with different system size give very similar results indicating that the system size is large enough compared to the phase field model interface thickness to ensure a good convergence of the model. The invariance with respect to the system size also indicates that the zeroth order expression of ref. [2] is sufficient to describe the perturbation of the stress intensity factors at the crack tip due to the kink. When turning to the results strictly speaking it is obvious that the trend of the phase field data is very similar to the one of the analytical prediction with a rather small discrepancy at low kink angles. The phase field data slightly overestimates the threshold value. Nevertheless, there is a significant overestimation of the increase in fracture energy induced by the kink, especially at angles above $30^{\circ}$.

Plotting the phase field profile in fig 4 gives a first hint of the origin of this discrepancy. One can see that on the obstacle side, the phase field profile is significantly steeper : the exponential tail is no longer present and in the 
interface region the profile is also much steeper. This translates into the fact that the fracture energy along the obstacle is higher than the fracture energy in the free propagation region. A numerical calculation of the fracture energy ${ }_{4}^{4}$ shows that there is a relative increase in it of approximately of 6 (resp. 17) percent for $\theta=20^{\circ}\left(\operatorname{resp} 40^{\circ}\right)$. Such values are consistent with the deviation from the prediction of eq. (14) which is also of 5 (resp. 16 ) percent. The fact that, as in 35] the crack profile is steeper close to the obstacle is clearly related to the change in the elastic energy density field at the crack tip induced by the crack kink. Indeed, when plotting the phase field contour line for $\varphi=0.5$, that is a good approximation of the crack profile together with the contour lines of the elastic energy density (multiplied by $g(\varphi)$ ), one can see in fig.2, that the maximum elastic energy density at the tip of arrested cracks is higher than at the tip of a freely propagating crack. It is also located closer to the boundary of the obstacle. As a result the local driving force toward $\varphi=0$ is higher on the obstacle side. This leads to a steeper profile (and an higher fracture energy). Hence the mode mixity at the crack tip induced by the imposed kink induces a change of the fracture profile, and as a consequence an unphysical increase of the fracture energy. In the well documented case $\theta=90^{\circ}$ 26, 13, 27, simulation results (now shown) indicate that the crack will propagate along the interface for $G>8 G_{c}$. This is much higher than the predicted threshold of $4 G_{c}$ and is expected since the effects of the crack profile steepenning are higher.

Here we have seen that when encountering an infinitely tough region the phase field crack behaviour is qualitatively correct. However some discrepancies with theoretical predictions are present due to a change in the fracture profile coming from the presence of the infinitely tough region. This gives confidence that the phase field model will be able to describe qualitatively well the crack front path in a complex heterogeneous material where one phase cannot be broken.

\subsection{Two regions of finite toughness}

This section describes what happens when a crack meets a region of finite toughness and more specifically it focuses on the effects of the choice of the mathematical description of the interface between the two regions. We consider a situation where the values of the load, and the fracture energy in both regions are such that the crack cannot propagate steadily in the region II. The results presented here have been obtained with $\Gamma_{I I}=2 \Gamma_{I}$, but the same results were obtained with higher ratios $\Gamma_{I I} / \Gamma_{I}$. The interface is described as a region of finite thickness $w_{\text {int }}$ over which the fracture energy goes from $\Gamma_{I}$ to $\Gamma_{I I}$ :

$$
\begin{aligned}
\Gamma(x, y) & =\Gamma_{I}+\left(1-\Gamma_{I I}\right)\left(1+\tanh \left(d / w_{\text {int }}\right)\right) / 2, \\
d & =\cos \theta\left(x-x_{o f f}\right)+\sin \theta(y) .
\end{aligned}
$$

\footnotetext{
${ }^{4}$ The fracture energy is estimated as $\cos (\theta) \int_{-W / 2}^{W / 2} d y \frac{D}{2}\left|\nabla_{2 D} \varphi\right|^{2}+h V(\varphi)-\varepsilon_{c}(g(\varphi)-1)$ in the region where the crack is supposed to follow the interface with the obstacle. The same formula with $\theta=0$ is used in the free propagation region.
} 

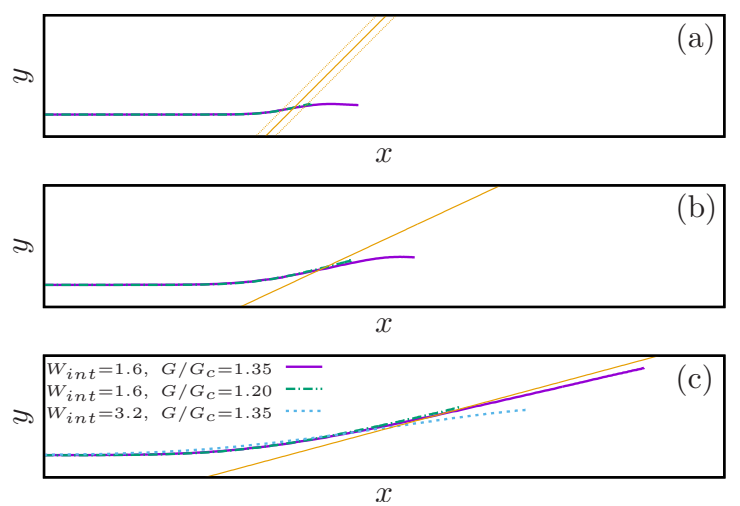

Figure 5: (a), (b), (c) Trajectories of the crack tip for $\theta=45,25$ and 15 degrees respectively. The thick orange line corresponds to the interface between the region I and II. In (a) two dotted orange lines are plotted 1.6 space units appart from the interface. The correspondence between line style and parameter values is given in (c). It is worth mentioning that the load is such that it corresponds to crack propagating along the interface in fig. 3

If the mathematical description of the interface between the two regions is correct, the crack propagation regime should be the same as in the previous section and the results should agree (at least qualitatively well) with the analytical prediction of eq. (14). In this framework, there are three control parameters: the angle $\theta$ and the applied load (that were already present in the previous section) and the interface thickness between the two phases that will be denoted $w_{\text {int }}$. The first two parameters are physically relevant and the behaviour of the crack should solely be a function of them while the latter is a model parameter that must be chosen properly to ensure that the crack motion is correct.

Before turning to the results, it is useful do discuss the the values of $w_{\text {int }}$ for which the model results can be expected to be correct. In the situations where the fracture energy is smoothly varying over lengthscales that are much larger than the fracture interface thickness, it has been shown that [12, 21] the phase field model is able to describe the crack path in good agreement with LEFM theory. In this situation, the force that pushes the crack path toward a kink is proportional to the fracture energy gradient while the force that pulls it back toward a straight path is proportional to the kink angle. As a result when $w_{\text {int }}>>w_{\varphi}$, the crack will, probably, move inside the boundary between the two domains with, possibly, a slight kink that will be a function of both $\theta$ and $w_{\text {int }}$ and it will eventually stop. This behaviour differs dramatically from what is predicted and observed when a crack meets a region of high toughness. Therefore, it is expected that the proper behaviour is observed when $w_{\text {int }}$ is small enough. In the following the reality of these expectations will be compared to actual numerical results and the implication of these results will be discussed.

To this end we first consider the large $w_{\text {int }}$ limit. In this case, as expected and for all values of the tilt angle taken between 10 and 45 degrees the crack, when hitting the interface keeps on propagating until the local fracture energy is large 


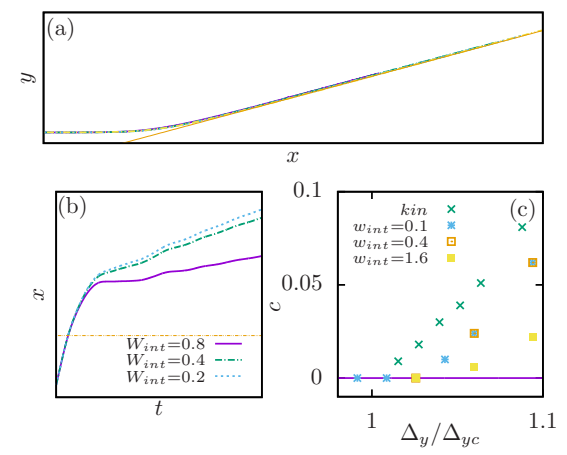

Figure 6: $\theta=15^{\circ}$ (a)Plot of the crack tip trajectories for different values of the interface thickness and a same value of load. (b) Plot of the $x$ position of the crack tip as a function of time for different different values of the interface thickness. (c) Plot of the crack velocity as a function of load for different values of the interface thickness. In (c) $\theta=10^{\circ}$.

enough to prevent crack propagation. Typical illustrations of this behaviour are shown fig (5) for angles 15, 25 and 30 degrees and different values of the applied load and two different values of $w_{\text {int }}$ that are of the same order of magnitude as the fracture interface thicknes: ${ }^{5}$. In all cases, the crack behaves as it were propagating in a smooth fracture energy landscape, which does not correspond to the behaviour expected when the crack is meeting an interface between two regions with different fracture energies.

We now turn to the case where $w_{i n t}$ is small. In this case, a deflection of the crack path approximately along the interface between the two regions is expected. In a first set of simulations with small values of $\theta \approx 10^{\circ}$ it is shown that this behaviour can be actually observed and the convergence of some measurable quantities is discussed. To this end we consider first a load value for which a crack was propagating along the interface in the infinitely tough region II case and consider different values of $w_{\text {int }}$. As can be seen in fig. 6 (a), for sufficiently small values of $w_{\text {int }}$ the crack path follows very well the interface and the trajectories are nearly indistinguishable. Nevertheless, when considering the position of the crack tip as a function of time, there is a marked difference between the two values of the crack velocity when it is travelling along the interface as can be seen in fig. 6.(b). In fig. 6(b) the results are summarized: the crack velocity is plotted against the applied load for different values of $w_{\text {int }}$. The plot indicates that there is a convergence toward a limiting value of the crack velocity when $w_{\text {int }}$ goes to zero. This limiting value is smaller than the one obtained in the kinetic case. This translates into a higher threshold for crack propagation along the interface and, as a result, into a higher discrepancy with the analytical predictions. Hence, when considering small values of $w_{\text {int }}$ and small tilt angles, the phase field model is able to reproduce qualitatively the

\footnotetext{
${ }^{5} w_{\text {int }}$ is 1.6 or 3.2 grid points while the fracture interface thickness is about 4 to 5 grid points as can be seen in fig 4
} 

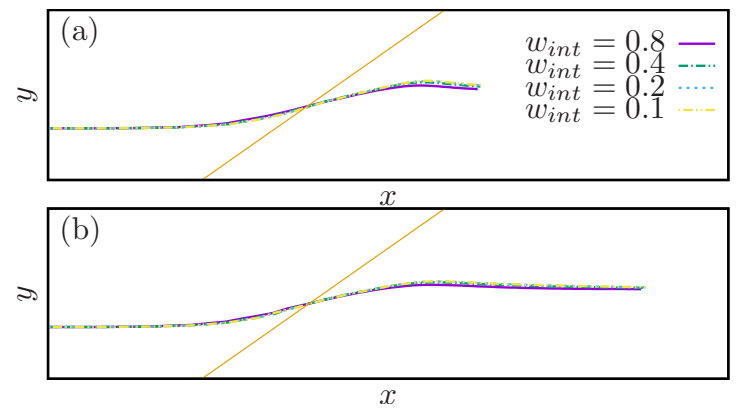

Figure 7: (a) Plot of the crack tip trajectories for $\theta=35^{\circ}$ and different values of the interface thickness. The load is such that $G / G_{c}=1.35$. In (b) the same is plotted with $G / G_{c}=1.43$. The load values are respectively below and above the threshold value obtained in the infinitely tough case presented in fig 3 (b).

results obtained using the LEFM. Nevertheless there is a significant quantitative discrepancy which is clearly related to the fact that the crack (defined as the region where $\varphi$ differs from 1 ) has entered the high energy region over at least one grid point due to the mode mixity that favours the propagation into the high fracture energy region. This results in an increase in the apparent fracture energy that is higher than the one due to the steepening of the $\varphi$ profile that was induced by the effects of both mode mixity and kinetic blockage.

Now we turn to the case where the kink angle is large. More specifically we consider values about 30 degrees. In this case, for all values of the load (up to values for which the crack can propagate in the region II) the crack does not propagate along the interface and the system always evolves toward a situation where a crack has entered (to some extent) the high fracture energy zone. For high values of $w_{\text {int }}$ typical paths are shown in fig. (5) while for low values a typical path is shown in fig. (6) In the latter case, a closer look at the crack profile together with the boundary between the two phases indicates clearly that, as previously, a significant amount of the diffuse crack is in the region II and therefore participates in the increase in apparent fracture energy and to the unphysical crack behaviour.

From this latter set of simulations it is clear that the phase field model used here with the naive description of fracture toughness inhomogeneities cannot describe properly crack propagation in an environment where the fracture energy varies abruptly. While for lower values of the kink angle the agreement could be described as qualitative, here, there is a strong qualitative difference that will dramatically affect the transition from interfacial to bulk cracks. This is due to the diffuse interface modelling of crack that implies that when a diffuse crack meets a heterogeneity, it will, regardless of the sharpness of the boundary between the two regions, feel the high energy region. Hence it is extremely likely that this result will extend to other approaches, including modelling approaches where the relaxational kinetics of the phase field used here is replaced by an actual minimisation of the free energy functional. 

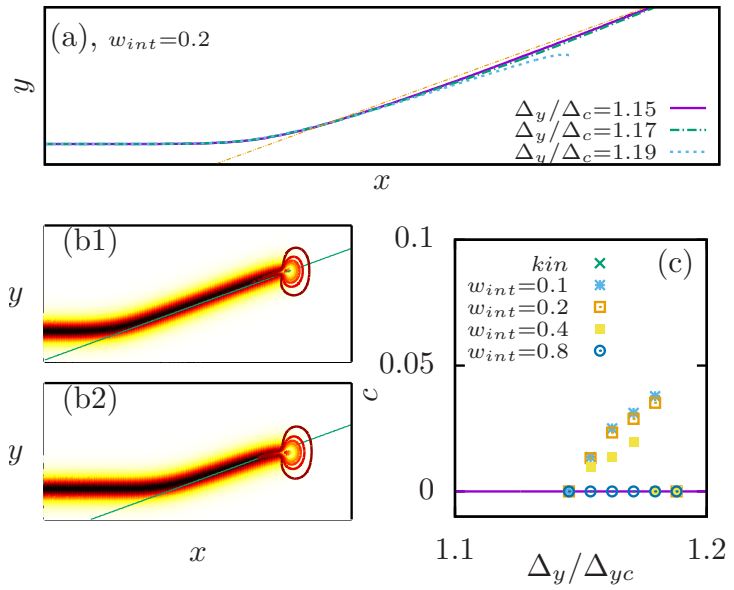

Figure 8: The value of $\theta$ is 20 degrees. (a) Plot of the crack tip trajectories, for three values of the load. For the two lower values, the crack is propagating along the interface while for the higher values (below the propagation threshold in region II) it goes through the interface and stops.(b1) and (b2), color plots of the phase field, the elastic energy density is plotted using a contour together with the position of the interface between region I and II. Here $w_{\text {int }}=0.1$. In b1 the load is $\Delta_{y}=1.15 \Delta_{c}$ while in b2 it is $\Delta_{y}=1.19 \Delta_{c}$. One can see that in both cases, a significant part of the crack is in the region II where fracture energy is high. (c) crack tip velocities along the interface as a function of the applied load for different values of the thickness of the interface between the two regions. The result from the previous section is also plotted as a reference. One should note that for high values of the load the crack stops for any value of $w_{\text {int }}$. 
For the sake of completeness, and to bridge the gap between these two limit cases, results obtained for intermediate values of $\theta$ is briefly discussed here. We consider the value $\theta=20^{\circ}$, and present simulation results obtained for small values of $w_{\text {int }}$. In fig. 8(a) trajectories of the crack for varying values of the applied load are presented. Surprisingly, when the applied load is increased two successive transitions are observed. At first the crack does not propagate as expected, and then above a load threshold it propagates. Finally above a second load threshold that is a function of the interface thickness the crack enters the region II and stops. It should be noted that however small is $w_{\text {int }}$, the second threshold is low as is illustrated in fig. 8(c) where the interfacial crack velocity is plotted against the load. Hence in the case of intermediate angles, a completely unphysical behaviour is also observed.

The reason for this is made clear when considering the crack, elastic energy density field and obstacle position as in fig 8 (b1, b2). Indeed, one can see that the crack interface is clearly inside the region II, which implies that the fracture energy is higher than the one in the region II. The fact that the crack has entered the region II is related to the mode mixity that is illustrated by the asymmetric repartition of the elastic energy density with respect to the axis of the kinked crack. Hence, the interplay between the diffuse interface representation of the crack and a heterogeneous fracture energy field prevents the phase field model from properly describing crack propagation when it could propagate in the different phases of a multiphasic material.

\section{Conclusion}

Here we have considered a simple setup to characterize how well a phase field model can describe the propagation of a crack in a heterogeneous material where the heterogeneities correspond to abrupt changes in the fracture energy. Simulations show that in the case of heterogeneities that are sufficiently tough to prevent crack propagation, a qualitative agreement with theoretical predictions made using LEFM. Moreover a quantitative comparison indicates that the small differences can be attributed to a steepening of the crack profile,

In the case where the fracture energy in the heterogeneity is finite but significantly larger than in the main region, the interplay between the diffuse crack and the boundary between the two regions implies significant changes in the apparent fracture energy that lead to unphysical behaviour. More specifically the mode mixity along the interfacial crack path implies that the crack will partially propagate in the high fracture energy region while the desired physical behaviour would be an interfacial crack propagating in the low fracture energy region. This, may give a rationale to recent numerical results in more complex systems such as clay composites 28. where it has been found that the material strength is decresing with the thickness of the weak interphase between hard silicates inclusion and the mattrix

The question of whether a not so naive description can be proposed is open. When the interface plays a significant role because its properties differ from bulk properties, alternatives have been proposed [29, 5]. They rely on the use of 
cohesive zone models to describe the interface failure and care has been taken to ensure a proper transition from bulk cracks to interfacial cracks and the added complexity to the plain phase field modelling is simply the translation of the actual complexity of the materials. However, in the case of fully bonded systems where the interface between the phases is a simple geometric object, a proper simple description of cracks propagation is still missing. Since the unphysical behaviour observed here is strongly related to the diffuse description of the crack an explicit description of the transition from one region to another region is likely to be needed.

\section{acknowledgments}

I am grateful to the referees of an earlier version of this manuscript for their

constructive criticisms. I also wish to thank A. Rowe for carefully checking the english of this manuscript.

\section{References}

[1] Najmul Abid, Mohammad Mirkhalaf, and Francois Barthelat. Discreteelement modeling of nacre-like materials: Effects of random microstructures on strain localization and mechanical performance. Journal of the Mechanics and Physics of Solids, 112:385-402, 2018.

[2] M. Amestoy and J. B. Leblond. Crack paths in plane situations-II. Detailed form of the expansion of the stress intensity factors. International Journal of Solids and Structures, 29(4):465-501, 1992.

[3] Jeremy Bleyer and Jean-François Molinari. Microbranching instability in phase-field modelling of dynamic brittle fracture. Applied Physics Letters, 110(15):151903, 2017.

[4] Blaise Bourdin, Gilles A. Francfort, and Jean Jacques Marigo. The variational approach to fracture. 2008.

[5] V. Carollo, J. Reinoso, and M. Paggi. Modeling complex crack paths in ceramic laminates: A novel variational framework combining the phase field method of fracture and the cohesive zone model. Journal of the European Ceramic Society, 38(8):2994 - 3003, 2018. Cermodel 2017: Modelling and Simulation Meet Innovation in Ceramics Technology.

[6] Chih-Hung Chen, Tristan Cambonie, Veronique Lazarus, Matteo Nicoli, Antonio J Pons, and Alain Karma. Crack Front Segmentation and Facet Coarsening in Mixed-Mode Fracture. Phys. Rev. Lett., 115(26):265503, 2015.

[7] F Corson, M. Adda-Bedia, H Henry, and E Katzav. Thermal fracture as a framework for quasi-static crack propagation. International Journal of Fracture, 158(1):1-14, jun 2009. 
[8] F Corson, H Henry, and M Adda. A model for hierarchical patterns under mechanical stresses. Philosophical Magazine, 90:1-18, 2010.

[9] A. G. Evans, Z. Suo, R. Z. Wang, I. A. Aksay, M. Y. He, and J. W. Hutchinson. Model for the robust mechanical behavior of nacre. Journal of Materials Research, 16(09):2475-2484, 2001.

[10] G.A. Francfort and J.-J. Marigo. Revisiting brittle fracture as an energy minimization problem. Journal of the Mechanics and Physics of Solids, 46(8):1319-1342, aug 1998.

[11] L B Freund. Dynamic Fracture Mechanics. Cambridge University Press (UK), 1990.

[12] Vincent Hakim and Alain Karma. Crack Path Prediction in Anisotropic Brittle Materials. Physical Review Letters, 95(23):235501, 2005.

[13] Ming Yuan He and John W. et al Hutchinson. Kinking of a crack out of an interface. Journal of Applied Mechanics, 56:270, 1989.

[14] H Henry. Study of the branching instability using a phase field model of inplane crack propagation. Europhysics Letters, 83:16004, 2008.

[15] H. Henry and M. Adda-Bedia. Fractographic aspects of crack branching instability using a phase-field model. Physical Review E, 88(6):060401, dec 2013.

[16] H Henry and H Levine. Dynamic instabilities of fracture under biaxial strain using a phase field model. Phys. Rev. Lett., 93(10):105504, 2004.

[17] Hervé Henry. Crack front instabilities under mixed mode loading in three dimensions. EPL (Europhysics Letters), 114(6):66001, 2016.

[18] M Z Hossain, C.-J. Hsueh, B Bourdin, and K Bhattacharya. Effective toughness of heterogeneous media. Journal of the Mechanics and Physics of Solids, 71:15-32, 2014.

[19] A Karma, D A Kessler, and H Levine. Phase-Field Model of Mode \{III $\}$ Dynamic Fracture. Phys. Rev. Lett., 87:45501, 2001.

[20] A Karma and A E Lobkovsky. Unsteady crack motion and branching in a phase-field model of brittle fracture. Phys. Rev. Lett., 92(24):245510, 2004.

[21] C. Kuhn and R. Müller. A discussion of fracture mechanisms in heterogeneous materials by means of configurational forces in a phase field fracture model. Computer Methods in Applied Mechanics and Engineering, 312:95 - 116, 2016. Phase Field Approaches to Fracture.

[22] Charlotte Kuhn, Alexander Schlüter, and Ralf Müller. On degradation functions in phase field fracture models. Computational Materials Science, 108:374 - 384, 2015. Selected Articles from Phase-field Method 2014 International Seminar. 
[23] Jean-Baptiste Leblond, Alain Karma, and Véronique Lazarus. Theoretical analysis of crack front instability in mode I+III. Journal of the Mechanics and Physics of Solids, 59(9):1872-1887, sep 2011.

[24] Bin Li, Daniel Millán, Alejandro Torres-Sánchez, Benoît Roman, and Marino Arroyo. A variational model of fracture for tearing brittle thin sheets. Journal of the Mechanics and Physics of Solids, 119:334-348, 2018.

[25] Junjie Liu, Wenqing Zhu, Zhongliang Yu, and Xiaoding Wei. Size effects in layered composites - Defect tolerance and strength optimization. Composites Science and Technology, 165:154-160, 2018.

[26] He Ming-Yuan and John W Hutchinson. Crack deflection at an interface between dissimilar elastic materials. International Journal of Solids and Structures, 25(9):1053-1067, 1989.

[27] N Moës, C Stolz, P Bernard, and N Chevaugeon. A level set based model for damage growth: The thick level set approach. International Journal For Numerical Methods in Engineering, 86(December 2010):358-380, 2011.

[28] Mohammed A Msekh, N H Cuong, G Zi, P Areias, X Zhuang, and Timon Rabczuk. Fracture properties prediction of clay / epoxy nanocomposites with interphase zones using a phase field model. Engineering Fracture Mechanics, 188:287-299, 2018.

[29] T T Nguyen, J Yvonnet, Q.-Z. Zhu, M Bornert, and C Chateau. A phasefield method for computational modeling of interfacial damage interacting with crack propagation in realistic microstructures obtained by microtomography. Computer Methods in Applied Mechanics and Engineering, 312:567-595, 2016.

[30] Thanh Tung Nguyen, Julien Réthoré, and Marie-Christine Baietto. Phase field modelling of anisotropic crack propagation. European Journal of Mechanics - A/Solids, 65:279-288, 2017.

[31] Antonio J Pons and Alain Karma. Helical crack-front instability in mixedmode fracture. Nature, 464(7285):85-89, mar 2010.

[32] 0. Ronsin, F Heslot, and B Perrin. Experimental study of quasistatic brittle crack propagation. Physical Review Letters, 75:2352-2355, 1995.

[33] 0. Ronsin, F Heslot, and B Perrin. Dynamics of quasistatic directional crack growth. Physical Review E, 58:7878-7886, 1998.

[34] O. Ronsin, C. Caroli, and T. Baumberger. Crack front échelon instability in mixed mode fracture of a strongly nonlinear elastic solid. EPL (Europhysics Letters), 105(3):34001, feb 2014. 
[35] Juan Michael Sargado, Eirik Keilegavlen, Inga Berre, and Jan Martin Nordbotten. High-accuracy phase-field models for brittle fracture based on a new family of degradation functions. Journal of the Mechanics and Physics of Solids, 111:458-489, 2018.

[36] Daniel Schneider, Ephraim Schoof, Yunfei Huang, Michael Selzer, and Britta Nestler. Phase-field modeling of crack propagation in multiphase systems. Computer Methods in Applied Mechanics and Engineering, 312:186195, 2016.

[37] E Tanné, T Li, B Bourdin, J Marigo, and C Maurini. Crack nucleation in variational phase-field models of brittle fracture. Journal of the Mechanics and Physics of Solids, 110:80-99, 2018.

[38] A Yuse and M Sano. Instabilities of quasi-static crack patterns in quenched glass plates. Physica D, 108:365-378, 1997. 